and albeit uncommon, remains a clinically significant cause of death with a mortality of up to 21.7 per 100,000 in the UK. In addition, $6 \%$ of pulmonary embolisms have an upper extremity source. We recognise the lack of official guidance on the diagnosis and management of CA-UEDVT. Herein, we aim to discuss the up-to-date management strategies for this prevalent condition.

Methods A systematic review was conducted according to PRISMA guidelines (search string: "catheter" AND "UEDVT") between 1997-2021 on PubMed. 53 manuscripts were screened by a single author (OA) on Rayyan and 50 were included in the final analysis. We extracted data on the institution, journal, citations, topic, sample size and outcomes.

Results Our results show that the commonest symptoms reported are ipsilateral upper extremity pain and/or discomfort, oedema, arm fatigue and discoloration at catheter entry site, but the majority of UEDVTs are asymptomatic. A high index of suspicion is therefore required.

For its diagnosis, venous duplex scan (VDS) is the commonest imaging modality used but it is less sensitive in paediatric patients and has limited use for central vasculature. In clinically suspected CA-UEDVT with negative VDS, contrast-venography - the gold-standard - can be used. It is, however, an invasive and technically complex procedure which requires use of contrast. For the management we recommend anticoagulation without removal of catheter for as long as catheter is in place and continued for at least three months after removal. (Low-molecular-weight-heparin in cancer patients and Direct-Oral-Anti-Coagulants in non-cancer patients unless contra-indicated). Consider removal if there is a catheter-associated infection, continuation of symptoms despite treatment and if the catheter is no longer functional or required. Finally, consider catheter-directed thrombolysis when indicated.

Conclusions Our findings provide a preliminary ground for further research into the diagnostic features of CA-UEDVT and may advise on the most up-to-date management algorithm for this condition.

\section{S81 10 YEAR RETROSPECTIVE AUDIT FROM AMBULATORY PULMONARY EMBOLISM PATHWAY IN A TERTIARY HOSPITAL}

R Sudhir, E Bailie, N Parmar. University Hospitals of Leicester NHS Trust, Leicestershire, UK

\subsection{6/thorax-2021-BTSabstracts.87}

Introduction The suspicion of PE (Pulmonary embolism) is a common reason for hospital admission and hospital stays. Being able to prevent admission in low risk PE patients has previously shown to be safe and cost effective with an established ambulatory service running in our hospital since 2010.

Methods A 10 year retrospective analysis from June 2010 to January 2020 was carried out using the PE database. Patients with suspected PE referred to the service using acceptance criteria (appendix a). PE risk was then stratified using the PE severity index (PESI). D-dimers were performed in the low and intermediate probability groups. Those with negative $\mathrm{d}$ dimers were discharged; those with high risk or positive ddimer underwent imaging in the form of CT pulmonary angiography (CTPA) or ventilation-perfusion (VQ) scanning. This was generally a same day service.
Results Total number of patients referred to the service was 6434. 2825 (43\%) were through bed bureau, $1491(23 \%)$ through Clinical decisions unit, 732 (11\%) though Emergency department and further $700(10.8 \%)$ through other services.

From the total number of 6434,3724 (58\%) did not require any scans and were safely discharged from the service. CTPulmonary angiogram (CTPA) was performed in 2126 (33\%) and Ventilation-Perfusion (V/Q) scans in 584 (9\%). 2710 (42\%) patients underwent scanning with 429 (15.8\%) positive and $2281(84 \%)$ were negative. 6112 (95\%) of these patients were managed as outpatients. All patients were contacted by nurse led telephone follow-up, 1 week post diagnosis. Consultant led follow-up was variable, approximately $74 \%$ (321/429), due to multi-consultant service.

Conclusions Outpatient management of Pulmonary embolism is a safe and effective service.

\section{S82 USING CARDIAC MAGNETIC RESONANCE IMAGING TO ASSESS CARDIAC GEOMETRY IN THE DIAGNOSIS OF CHRONIC THROMBOEMBOLIC DISEASE AND CHRONIC THROMBOEMBOLIC PULMONARY HYPERTENSION}

${ }^{1} \mathrm{M}$ McGettrick, ${ }^{1} \mathrm{H}$ Dormand, ${ }^{1} \mathrm{M}$ Brewis, ${ }^{2} \mathrm{NN}$ Lang, ${ }^{1} \mathrm{M}$ Johnson, ${ }^{1} \mathrm{AC}$ Church. ${ }^{1}$ Scottish Pulmonary Vascular Unit, Glasgow, UK; ${ }^{2}$ University of Glasgow, Glasgow, UK

\subsection{6/thorax-2021-BTSabstracts.88}

Background Cardiac magnetic resonance (CMR) imaging is the gold standard tool for evaluating the right ventricle (RV) in chronic thromboembolic disease (CTED) and chronic thromboembolic pulmonary hypertension (CTEPH). Ventricular septal flattening, reflecting RV pressure overload in idiopathic pulmonary arterial hypertension (IPAH) has been quantified using the eccentricity index (EI) with echocardiography and pulmonary artery distensibility (PAD) has been shown to correlate with pulmonary artery pressure. These have not been evaluated for use in CTEPH using CMR. We assessed them in the detection of CTED and CTEPH and correlated with haemodynamics.

Methods CMR and right heart catheterisation were performed on 30 patients with CTEPH and 20 sex-matched controls without resting pulmonary hypertension (10 patients with no thrombotic disease and 10 with CTED) at a national pulmonary hypertension centre. Mid-papillary short axis view was used to assess the eccentricity index at end-systole and enddiastole. Main PAD was measured using velocity-encoded CMR, perpendicular to pulmonary artery.

Results EI at end-systole and end-diastole were significantly increased in CTEPH compared to controls (1.3 (0.5)vs1.0 (0.01); $\mathrm{p} \leq 0.01$ and (1.22 (0.2)vs0.98 (0.01); $\mathrm{p} \leq 0.01$, respectively). PAD was significantly reduced in CTEPH compared to controls $(0.13(0.1)$ vs $0.46(0.23) ; \mathrm{p} \leq 0.01)$. End-systolic EI and end-diastolic EI significantly correlated with pulmonary vascular haemodynamic indices, including mean pulmonary arterial pressure, cardiac output and with NTproBNP. End-systolic and End-diastolic EI correlated with exercise capacity as measured by 6-minute walk distance, and with pulmonary artery distensibility (R-value 0.8 ). Using ROC curves, an optimal threshold of 1.1 for both end-diastolic and end-systolic indices identified the presence of pulmonary hypertension. Both EI and PAD were able to differentiate the presence of CTED from normal. 
A

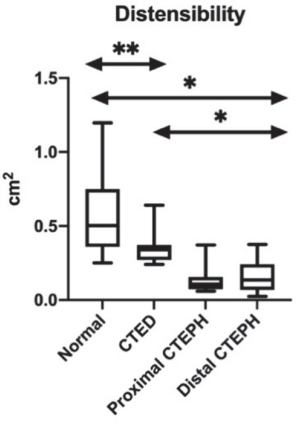

Diastolic LV Eccentricity Index

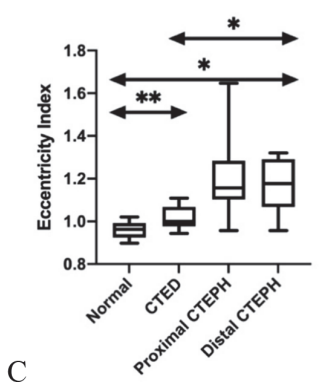

Systolic LV Eccentricity Index

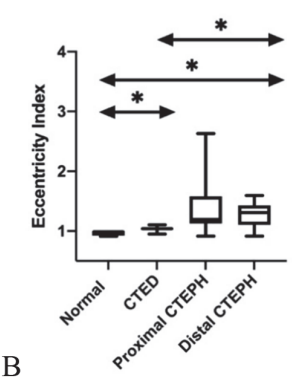

Abstract S82 Figure $1 \mathrm{MRI}$ indices in patients with no pulmonary vascular obstruction, CTED, proximal and distal CTEPH

P-value $=$ ANOVA

${ }^{*} p=<0.01{ }^{* *} p=<0.05$, ns $=$ not significant

A - Pulmonary Artery Distensibility, B - Systolic Left Ventricular EI, C Diastolic Left Ventricular El

Conclusion EI and PAD correlate with invasive haemodynamic indices and right ventricular function in CTEPH. These measures strengthen the ability of CMR to detect pulmonary hypertension and provide further justification for the use of CMR in investigation of chronic thromboembolic disease.

\section{Biologics for asthma}

\section{S83 THE IMPACT OF AN ONLINE PATIENT-FACING TOOL ON SEVERE ASTHMA REFERRALS}

C Renwick, C Cheung, A Fallas, J Kirby, S Walker. Asthma UK, London, UK

\subsection{6/thorax-2021-BTSabstracts.89}

Introduction Biologics for severe asthma (SA) are effective but only $18 \%$ of people with suspected SA are referred for consideration for treatment as recommended by current BTS asthma guidelines. ${ }^{1}$ Awareness of biologics among people with asthma is low. Here we evaluate the impact of a patient-facing intervention designed to drive appropriate SA referrals in the UK.

Methods Asthma UK (AUK) designed an interactive online tool that directs people to one of 5 possible outcomes, including 'improve asthma management', 'seek referral' or 'explore biologic options'. Each outcome has a clear call to action and option to sign up for tailored health advice emails. The tool was developed based on the Asthma Control Questionnaire (ACQ) and service specification for SA. The tool was created using an online survey platform (Typeform) and embedded on the AUK website. Usability of the tool was evaluated via moderated online video interviews with 12

patients with asthma. Respiratory clinicians were also invited to use the tool and provide feedback. It was promoted to people with uncontrolled asthma via AUK emails, social media and within the press. A follow-up survey was administered six weeks later to understand how people acted on the advice provided.

Results As of April 2020, 20,000 people had used the interactive tool. Of those that signed up for the tailored email, 313 completed the 'improve asthma management', 'seek referral' or 'explore biologic options' follow-up survey. Overall, 70\% (218/313) said they took action based on their outcome from the tool. Of those completing the 'seek referral' survey, 60\% (55/91) said they spoke to their GP about a referral and 30\% (27/91) were referred. Of those completing the 'explore biologic options' survey, 39\% (15/38) spoke to their healthcare professional about their eligibility and $21 \%$ (8/38) said they were assessed for treatment with biologics. Of those completing the 'improve asthma management' survey, $80 \%(148 / 184)$ said they took steps to improve their asthma management.

Conclusion Preliminary results suggest that AUK's interactive patient facing tool helps to increase SA referrals and access to biologic treatments.

\section{REFERENCE}

1. Asthma UK, Living in Limbo: the unmet need in difficult and severe asthma, 2019.

\section{S84 LONG-TERM EFFICACY OF DUPILUMAB: 3-YEAR DATA OF QUEST PATIENTS WITH MODERATE-TO-SEVERE ASTHMA ENROLLED IN LIBERTY ASTHMA TRAVERSE}

${ }^{1}$ ID Pavord, ${ }^{2} \mathrm{~A}$ Papi, ${ }^{3} \mathrm{~A}$ Bourdin, ${ }^{4} \mathrm{H}$ Watz, ${ }^{5} \mathrm{C}$ Domingo, ${ }^{6} \mathrm{ME}$ Wechsler, ${ }^{7} \mathrm{X}$ Mao, ${ }^{8} \mathrm{~B}$ Ortiz, ${ }^{9} \mathrm{M}$ Djandji, ${ }^{10} \mathrm{~L}$ Mannent, ${ }^{7} \mathrm{E}$ Laws, ${ }^{8} \mathrm{~N}$ Amin, ${ }^{8} \mathrm{DJ}$ Lederer, ${ }^{9} \mathrm{M}$ Hardin. ${ }^{1} \mathrm{NIHR}$ Oxford Biomedical Research Centre, University of Oxford, Oxford, UK; ${ }^{2}$ Respiratory Medicine Unit, University of Ferrara, S. Anna University Hospital, Ferrara, Italy; ${ }^{3}$ University of Montpellier, Montpellier, France; ${ }^{4}$ Pulmonary Research Institute, LungenClinic Grosshansdorf, Airway Research Center North (ARCN), German Center for Lung Research (DZL), Grosshansdorf, Germany; ${ }^{5}$ Corporació Sanitària Parc Tauli, Sabadell, Autonomous University of Barcelona, Barcelona, Spain; ${ }^{6}$ National Jewish Health, Denver, CO, USA; ${ }^{7}$ Sanofi, Bridgewater, NJ, USA; ${ }^{8}$ Regeneron Pharmaceuticals, Inc., Tarrytown, NY, USA; ${ }^{9}$ Sanofi, Cambridge, MA, USA; ${ }^{10}$ Sanofi, Chilly-Mazarin, France

\subsection{6/thorax-2021-BTSabstracts.90}

Introduction and Objectives Dupilumab, a fully human monoclonal antibody, blocks the shared receptor component for interleukin-4 and interleukin-13, key and central drivers of type 2 inflammation in multiple diseases, including asthma. In the phase 3 LIBERTY ASTHMA QUEST study (NCT02414854), add-on dupilumab 200/300 mg every 2 weeks (q2w) significantly reduced severe asthma exacerbations and improved pre-bronchodilator forced expiratory volume in 1 second $\left(\mathrm{FEV}_{1}\right)$ vs matched placebo in patients with uncontrolled, moderate-to-severe asthma. The LIBERTY ASTHMA TRAVERSE open-label extension study (NCT02134028) evaluated the long-term safety, tolerability, and efficacy of dupilumab in patients who had completed a previous dupilumab asthma study. This post hoc analysis evaluated the efficacy of dupilumab in patients from QUEST who rolled over into TRAVERSE and received a further 96 weeks of dupilumab treatment.

Methods We evaluated data from QUEST patients treated with dupilumab $\mathrm{q} 2 \mathrm{w}$ or matched placebo for 52 weeks who rolled over into TRAVERSE and received 96 weeks of dupilumab 